\title{
Psychological Impact, Early Behavioural Response to COVID-19 and Predictors Among Health Science Students in Amhara Region, Northwest Ethiopia
}

\author{
Agerie Mengistie Zeleke ${ }^{1, *}$, Gashaw Melkie Bayeh ${ }^{2}$ \\ ${ }^{1}$ Department of Midwifery, School of Public Health, Teda Health Science College, Gondar, Ethiopia \\ ${ }^{2}$ Department of Environmental Health, School of Public Health, Teda Health Science College, Gondar, Ethiopia \\ Email address: \\ ageriemengistie@gmail.com (A. M. Zeleke),megashaw21@gmail.com (A. M. Zeleke) \\ ${ }^{*}$ Corresponding author
}

To cite this article:

Agerie Mengistie Zeleke, Gashaw Melkie Bayeh. Psychological Impact, Early Behavioural Response to COVID-19 and Predictors Among Health Science Students in Amhara Region, Northwest Ethiopia. European Journal of Preventive Medicine. Vol. 9, No. 5, 2021, pp. 119-128. doi: 10.11648/j.ejpm.20210905.12

Received: September 3, 2021; Accepted: September 24, 2021; Published: October 5, 2021

\begin{abstract}
Background: Psychological impact of the Coronavirus 2019 (COVID-19) pandemic is a serious mental health problem for everyone, including health science college students who spend most of their times at home due to the closure of colleges. Addressing needs of early behavioral responses to this pandemic virus in the affected community particularly for students are very important. However, In Ethiopia, there is a paucity of evidence on the addressing psychological impact, early behavioral response to COVID-19. Therefore, this study aimed to assess the status of psychological impact, early behavioral response to COVID-19 and its associated factors among public health science college students, northwest, Amhara national regional State Ethiopia. Methods: An institutional-based cross-sectional study was employed among 317 respondents from October 16, 2020 to December 30, 2020. A systematic random sampling technique used to select the students who attending in public health science colleges in Amhara Regional state. Data were collected using an interviewer-administered structured questionnaire. Data were collected using an interviewer-administered structured questionnaire. Epi-data version 4.6 and SPSS version 20.0 software were used for data entry and analysis, respectively. Binary logistic regressions (Bivariable and multivariable) were performed to identify statistically significant variables. Adjusted odds ratio with $95 \%$ CI was used to declare statistically significant variables on the basis of $\mathrm{p}$-value $<0.05$ in the multivariable logistic regression model. Results: The overall status of psychological impact and early behavioral response to the COVID-19 pandemic among participants were $51.4 \%$ with $95 \% \mathrm{CI}(46 \%-57 \%)$ and $51.1 \%$ with $95 \% \mathrm{CI}(45 \%-57 \%)$ respectively. Age $18-24$ years old (AOR: 3.6; (CI: $1.14-$ 11.39)), urban residence (AOR: 0.51; 95\% CI: (0.31-0.84)), being male participants (AOR: 1.72, 95\% CI95\% CI: (1.04-2.84)), participants evolved in Debre Tabor health science college (AOR: 4.25; CI 95\% (1.60-7.72)) were significant predictors of psychological impact of COVID-19, whereas being female participants (AOR: 3.4; 95\% CI: (1.95-5.14)), and participant had psychological impact ofCOVID-19 (AOR: 1.9; 95\% CI: (1.16-2-96)) were significant factors with early behavioral response to COVID-19. Conclusions: The psychological impact of COVID-19 was higher among diploma students and early behavioral responses to prevent COVID-19 were not satisfactory. Hence, it is crucial track to early responses to the COVID-19 preventive measures, through appropriate information outlets, mainstream media, and relying on updating information from TV, radio, and health care workers about COVID-19 provision of behavioral readiness to COVID-19 prevention are very important. The government should implement national programs for school based health and safety, promoting students' psychological wellbeing.
\end{abstract}

Keywords: COVID-19 Pandemic, Early Behavioral Response, Psychological Impacts 


\section{Introduction}

The Coronavirus Disease 2019 (COVID-19) is a viral pandemic that first time reported in Wuhan, China, in December 2019 and has spread all over the world [1]. Then, WHO declared this novel COVID-19 as a pandemic disease on March 11, 2020, and a public health emergency of international concern because of poses a high risk to countries with vulnerable health care systems [2]. Now COVID-19 pandemic has became a major concern for global health and registered as the largest outbreak of novel corona virus $(2019-\mathrm{nCoV})$ or the severe acute respiratory syndrome corona virus 2 (SARS-CoV-2), since it rapidly spread from Wuhan, city province of China to the rest of the world [1]. The COVID-19 pandemics officially recognized in Ethiopia on 13 March 2020, after the Japanese arrived in Ethiopia from Burkina Faso trip, and tested positive for the novel COVID-19 [3].

According to case-fatality current reports, more than 2.5 million people have been getting infected, and more than 1.3 million deaths caused by corona virus globally [4] and the figures are still increasing rapidly almost all African countries have confirmed cases and the number of deaths is increasing [5-7].

The COVID-19has shown different common symptoms, such as fever (not in all), dry cough, sore throat, headache, fatigue, headache, myalgia, shortness of breath, sore throat and conjunctivitis have been described, but severity characters range from mild to severe in individuals [8-10].

The World Health Organization (WHO) has designed different mechanisms to combat the spread of COVID-19 outbreaks in public health aspects, such as hand washing with soap or use of hand sanitizers, maintaining social distance and home stay to remain protected from the COVID-19 infection [11-13]. However, there is an inadequate number of hospitals, shortages in transportation, lack of personal protective equipment (PPE), sanitizer, masks and early psychological readiness, low community awareness and strong enforcement to decrease the initiated a screening program, established quarantine and treatment centers, of the virus in developing countries [14].

The Ethiopian ministry of health also designed an emergency task force was done the spread of the COVID-19 pandemic may be prevented by staying at home, social distancing, wearing masks, and applying hand hygiene, alongside city lockdown facilities (college, university and schools), early detection, prompt treatment, and the implementation of a robust system to trace contacts $[8,15]$. The major challenge of this designed an emergency task force did not perceive the impact COVID-19 of mental health and the advantage of early behavioral response for this pandemic virus to peoples [16]. Since, identifying psychological wellbeing across countries and early response to the spread of the COVID virus were very necessary to compact impact of the COVID virus on humankinds [17].

On the other hand, complicated means of COVID-19 pandemic prevention have affected social mental health have psychosocial impacts on people. This is particularly serious for students, because college's student spends their times overlooked at home due to the closure of schools and they don access to information [18, 19]. If mental health complications are not well managed and make psychosocial readiness, it leads to the psychological distress like; fear, anxiety, suicidal attempts and negatively inhibits the learning process among the higher-risk groups of students [20, 21]. These showed that the COVID-19 outbreaks could affect mental health problems in the younger age groups students like; suicide, depression, distress, loss of their job, fear, worry, nervousness, and anxiety [22-24].

These provoking massive health measures to control the spread of the COVID-19 outbreak had a strong influence on the psychological impact, the economic social effect on the students, while separation from loved friends, educational system, the loss of freedom, staying in their homes, and uncertainty over disease status created dramatic adverse effects on mental health's $[25,26]$. Since communities have get a lot of fake information through un scientific sources that might be potentially disturb the public behavioral response [27]. These tensions are further magnified by fear of being quarantined or having to deal with inadequacies of personal protective equipment and ventilators [28]. This showed that some health workers have described feeling coerced and trying in outbreak situations [29].

The prevalence of mental health threats to human beings may occur as a result of closed colleges, overlooked and detachment of daily learning activities without early behavioral response from this mental complication [30, 31]. In particularly, students, psychological readiness is very important to the early behavioral response towards the COVID-19 pandemics as emergency health measurements [32]. The other institutional based study in India stated that symptoms of the psychological impact of COVID-19 on participants were an increase in the symptoms of stress, anxiety, and more socially withdrawn or feelings of self-harm of students [33].

Previous studies revealed that age, marital status, and sex, internet access, fake information, protective measures are available, students were significantly associated with the psychological impact of COVID-19 [29, 33-36]. Similarly, age, residence, academic performance, year of study, and social support, level of awareness of COVID-19 was determinants of early behavioral response to prevent COVID-19 [37-39].

The Ethiopia ministry of education has a plane to reopen schools, colleges and universities after students stayed at home for 6 months without evidence of the status of psychological impact and early behavioral response to compact COVID-19 pandemics on students. Investigating the student psychological impact and understanding early behavioral response preventive measures of the COVID-19 pandemic are very important.

Therefore, determine the status of psychological impact, and early behavioral response to COVID-19 and identify their associated factors among health science college students 
in Amhara regional state, Ethiopia. The findings of this study will help to design an effective intervention for the psychological impact and to make readiness to enhance behavioral response to COVID-19.

\section{Methods}

\subsection{Study Setting}

The study was conducted in the northwest of Amhara regional state, governmental health Science Colleges. It's located in Amhara Regional State, and is $748 \mathrm{~km}$ far from northwest of Addis Ababa, the capital of Ethiopia. There are five governmental health science colleges in Amhara Regional State. Of these, three governmental health Science Colleges are found in the northwest part of Amhara regional state. As Amhara regional health bureau reported, in three public health science collages' the total 30035 graduates student every year, of which 1468 are males and 1567 are female students.

\subsection{Study Design and Study Period}

An institutional-based cross-sectional study was conducted from October 16 to December, 30, 2020 to assess the prevalence and determinants of the psychological impact and to enhance early behavioral response to COVID-19 among students in public health science colleges in the northwest, Amhara Region, Ethiopia.

\subsection{Study Population and Eligibility Criteria}

We included all students in the public health science colleges' northwest, Amhara region, Ethiopia. All selected students who attended in public health Science College, during the data collection period were included. Students, who were unable to respond, seriously ill were excluded from the study.

\subsection{Sample Size Determinations and Sampling Procedures}

The sample size was determined by using single population proportion formula based on the following assumptions: A 95\% confidence level (1.96), the prevalence of psychological impact experienced by subjects during the last four weeks preceding the survey $(\mathrm{P}=25 \%)$ which was taken from the previous study conducted in Ethiopia [32], 5\% marginal error. The final sample size was 317 students, including a $10 \%$ non-response rate. In the northwest, Amhara regional state, there are three public health science colleges. Participants in all health science colleges were evolved to study. Proportion to size allocation was made to determine the required sample size for each health science college based on the average number of students at each college in the month proceeding the data collection period. Therefore, the allocated sample size from Teda Health Science College (103), Bahir Dar Health Science College (105), and Debre Tabor Health Science College (109). Lists of students from each the college's department were taken to prepare a sampling frame.

A systematic random sampling technique was used to select study participants in each of the health science colleges. The sampling interval $\mathrm{K}^{\text {th }}$ value for each health science college was calculated by dividing the source population by the total sample size. Therefore, the sampling third interval was used in all health science colleges to select study respondents.

\subsection{Study Variables and Data Measurements}

The dependent variables were the psychological impact of COVID 19 and its early behavioral response among public health science students. The independent variables included socio-demographic variables such as age, gender, marital status, profession, and current place of residency of students. Data were collected using an interviewer-administered structured questionnaire. The questionnaire was adapted from Google, prepared in English for an online survey then translated into Amharic (local language) and then back to English to ensure consistency. Nine diploma holder female midwives as data collectors and one BSc holder midwife as supervisor were recruited for the data collection process. The data collectors and the supervisor practiced all the necessary precautions, such as practicing hand hygiene, physical distancing, and wearing a face mask to avert the risk of acquiring the infection from the study participants.

The psychological impact of the COVID-19 pandemic and its early behavioral responses among health science college students were measured using questions adapted from the WHO survey tool [14] and guidance [40]. The mean score value was used to determine the level of psychological impact and early behavioral responses among students. Students' level of psychological impact was categorized as low and high psychological impact based on the mean score value which was $16.6 \%$. Therefore, a high psychological impact was reported when the students' mean score value was $\geq 16.6 \%$ otherwise students had a low psychological impact. Students' early behavioral response to the COVID-19 was also categorized as poor and good early behavioral responses based on the mean score value which was $13.6 \%$. Good early behavioral responses were reported when student's mean scores value was $\geq 13.6 \%$ otherwise students had poor early behavioral responses.

\subsection{Data Collection Tools and Procedures}

Data regarding socio-demographic, information exposure, risk perception of COVID-19, and precaution measures adaptation and students' early behavioral response to COVID-19 were collected through a face-to-face interview using a structured questionnaire adapted from different literature. Respondents were asked about the sources of information to COVID-19 and how much they trusted those sources. They were also asked about the types of information that they wanted to receive. Participants were interviewed about whether they performed precautionary measures including avoiding handshaking, adopting hand washing, and 
practicing physical distancing.

Data were collected by BSc midwifes and strictly followed by supervisors who managed the overall data collection process. All responses to closed and open questions were written down manually by the interviewers. The supervisors assessed the consistency and completeness of the data on a daily basis.

\subsection{Data Analysis}

Data were entered into Epi- Data version 4.6and then exported to SPSS version 20.0 software for analysis. Frequency tables were used to summarize demographic characteristics and prevalence of psychological impact. Bivariate logistic regression was performed separately for each independent variable. Independent variables with a pvalue $<0.25$ were entered into the final model for multivariable analysis. Variables in the mutually adjusted multivariable model with a two-sided $p$-value $<0.05$ were considered statistically significant. Crude and adjusted odds ratios with $95 \%$ CI were computed to assess the level of association between dependent and independent variables.

\subsection{Data Quality Management}

To assure the quality of the data, the tool was prepared first in English and then translated into the local language (Amharic) by language experts in English and Amharic languages for consistency. A one-day training was given to data collectors and supervisors on the objective of the study, tool, and how to collect the data by the principal investigators. A pretest was done before the actual data collection period at Blue Naile Health Science College. Appropriate modifications such as unclear questions and ambiguous wording, changing terms, rephrasing for better understanding, deleting, and adding some information for clarity were made to the tool accordingly. Data collection was closely monitored by investigators and supervisors. Each data collector checks the questionnaire from each study participant for completeness daily to ensure data quality. Each questionnaire was reviewed and checked for completeness daily by the supervisors and principal investigators. Hosmer and Leme-show goodness- of -fit test was used to check model fitness.

\section{Results}

\subsection{Socio-demographic Characteristics}

A total of 317 students participated in the study with a response rate of $100 \%$. Of all participants, the majority $(60.6 \%)$ of them were male. One hundred thirty four $(42.2 \%)$ student's age were found to be the range of 18-24 years old. Regarding marital status, more than half of the students who participated in the study were single. Of all study participants, $58.0 \%$ of them were rural residents, and $34.7 \%$ of them were earned families a monthly income $<1500$ Ethiopian Birr (Table 1).
Table 1. Socio-demographic characteristics of psychological impact and early behavioral response to COVID-19 pandemics among health science college students Amhara state of Ethiopia, 2020, $(N=317)$.

\begin{tabular}{|c|c|c|c|}
\hline Variables & Catagory & Frequency & Percent (\%) \\
\hline \multirow{4}{*}{ Ages } & $18-24$ & 134 & 42.2 \\
\hline & $25-31$ & 82 & 25.9 \\
\hline & $32-38$ & 64 & 20.2 \\
\hline & $\geq 39$ & 37 & 11.7 \\
\hline \multirow{2}{*}{ Sex } & Male & 192 & 60.6 \\
\hline & Female & 125 & 39.4 \\
\hline \multirow{4}{*}{ Marital status } & Single & 180 & 58.8 \\
\hline & Married & 86 & 27.1 \\
\hline & Divorced & 42 & 13.2 \\
\hline & Windowed & 9 & 2.8 \\
\hline \multirow{4}{*}{ Religion } & Orthodox & 150 & 47.3 \\
\hline & Muslim & 79 & 24.9 \\
\hline & Protestant & 47 & 14.8 \\
\hline & Catholic & 41 & 12.9 \\
\hline \multirow{7}{*}{ Department } & Laboratory & 67 & 21.1 \\
\hline & Environment & 61 & 19.2 \\
\hline & Pharmacist & 53 & 16.7 \\
\hline & Nurse & 44 & 13.9 \\
\hline & HIT & 37 & 11.7 \\
\hline & Health excitation & 32 & 10.1 \\
\hline & Midwife & 23 & 7.3 \\
\hline \multirow{3}{*}{$\begin{array}{l}\text { Type of study } \\
\text { area }\end{array}$} & Teda HSC & 103 & 32.5 \\
\hline & Debre tabor HSC & 105 & 35.1 \\
\hline & Bahir Dar HSC & 109 & 34.4 \\
\hline \multirow{2}{*}{ Residence } & Rural & 184 & 58 \\
\hline & Urban & 133 & 42 \\
\hline \multirow{4}{*}{ Family income } & $<1500$ & 110 & 34.7 \\
\hline & $1501-2500$ & 98 & 30.9 \\
\hline & $2501-3500$ & 73 & 23.0 \\
\hline & $>3500$ & 36 & 11.4 \\
\hline
\end{tabular}

\subsection{Psychological Impact of the COVID-19 Pandemics}

The findings demonstrated that, more than half of the study participants $(51.4 \% 95 \%$ CI: $(46 \%, 57 \%))$ had high psychological impact to COVID-19 pandemics (figure 1).

Among prevention methods the majority of them 286 (90.2\%) had got information from social media about COVID-19 pandemics. Among this, $81.7 \%$ heard from health care providers. Of all students, $55.8 \%$ of them had proper hand washing practice with water and soap, $71.9 \%$ of had perceived that avoiding touching nose, eye, and face with unwashed hands, and about $60.9 \%$ of them feel confident always covering mouth and nose during coughing and sneezing with bend elbow made them safe from COVID 19 infection.

About more than $59.9 \%$ of the respondents reported that maintaining at least 2-meter physical distance between individuals can protect them from acquiring COVID-19 infection and $90 \%$ of them had the skills to adhere recommended hand washing procedure to prevent COVID-19 
infection (Table 2).

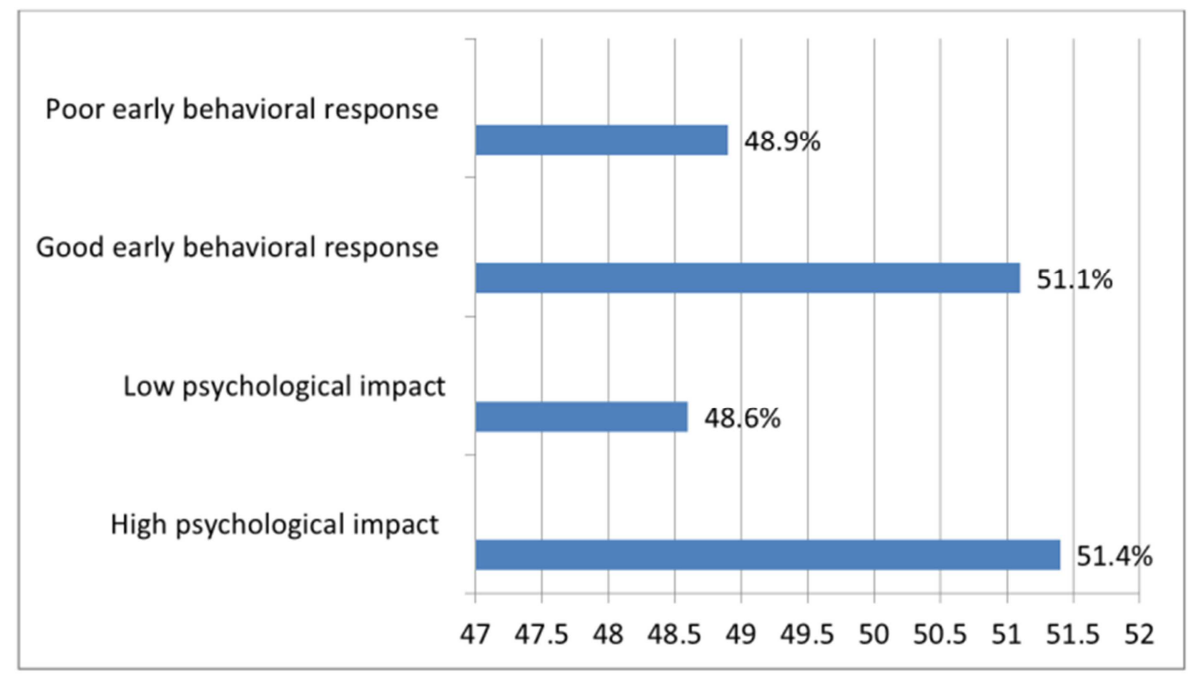

Figure 1. Prevalence of psychological Impact and early behavioral response to COVID-19 Pandemic.

Table 2. Frequency distribution of psychological impact towards COVID-19 pandemics among health science college students Amhara state of Ethiopia, 2020 $(N=317)$.

\begin{tabular}{lll}
\hline Had psychological impact of COVID-19? & Yes (\%) & No (\%) \\
\hline Getting information from social media? & $286(90.2$ & $31(9.8)$ \\
Getting information from health care workers? & $259(81.7)$ & $58(18.3)$ \\
Confident that can wash my hands frequently with soap and sanitizer? & $177(55.8)$ & $140(44.2)$ \\
Have the resource (water, sanitizer and soap) to wash my hands? & $201(63.4)$ & $116(36.6)$ \\
Confident that I can stay at home easily to prevent COVID-19? & $182(57.4)$ & $135(42.6)$ \\
Confident that I can save from crowed places and close contact? & $175(58.4)$ & $132(41.6)$ \\
Always cover during coughing using the bend of my elbow? & $228(71.9)$ & $142(44.8)$ \\
Avoid touching my eyes, nose and mouth to prevent infection of COVID-19? & $190(59.9)$ & $89(28.1)$ \\
Maintain at least 2-meter physical distance to other individuals & $259(81.7)$ & $127(40.1)$ \\
Believing that COVID19 is extremely non cure & $204(64.4)$ & $58(18.3)$ \\
Have confidence taken preventive measures to prevent COVID-19 infection & $148(46.7)$ & $113(35.6)$ \\
Concerns about other family members a getting COVID-19 & & $168(53.3)$ \\
\hline
\end{tabular}

\subsection{The Early Behavioral Response to COVID-19 Pandemics}

The findings of this study indicated that more than half of the study participants (51.1\%) 95\% CL: $(45 \%, 57 \%))$ had good early behavioral response to COVID-19 pandemics. More than two-thirds $63.4 \%$ of students were covering their mouths when coughing and sneezing and $88.6 \%$ of them had washed their hands properly with soap and water more often than usual time to reduce the spread of COVID 19. A majority, $88.0 \%$ of students avoiding sharing their utensils to prevent transmission of the virus although $60.3 \%$ of them did not wear face masks regardless of the presence or absence of symptoms of COVID 19. About $41.6 \%$ of the students were still going to crowded public transport /places and about $68.5 \%$ of them had stayed at home due to lockdown prevention standards. About $61.1 \%$ of the participants did not maintain better indoor ventilation in the health care system, while $67.8 \%$ of students had experienced good ventilation in their house to prevent COVID-19 transmissions (Table 3).

Table 3. Prevalence of early behavioral response to COVID-19 pandemics among health science college students, Amhara state, Ethiopia, 2020 (N=317).

\begin{tabular}{lll}
\hline Early behavioral response & Yes (\%) & No (\%) \\
\hline Is covering mouth when coughing and sneezing? & $201(63.4)$ & $116(36.6)$ \\
Avoiding sharing of utensils during means? & $279(88.0)$ & $38(12.0)$ \\
Do you wash hands with soap and water more often than usual? & $281(88.6)$ & $36(11.4)$ \\
Do wash immediately after coughing, rubbing nose, sneezing? & $261(82.3)$ & $56(17.7)$ \\
Washing hands after touching contaminated objects and patients? & $205(64.7)$ & $112(35.3)$ \\
Wearing mask regardless of the presence or absence of symptoms? & $126(39.7)$ & $191(60.3)$ \\
Do avoid eating outside on café or hotel? & $100(31.5)$ & $217(68.5)$ \\
Do you avoid public places / transport? & $132(41.6)$ & $185(58.9)$ \\
Have you maintain better indoor ventilation on health care? & $102(32.2)$ & $215(67.8)$ \\
Do you increase surface cleaning in health care? & $266(83.9)$ & $51(16.1)$ \\
\hline
\end{tabular}




\subsection{Factors Associated with Psychological Impact of the COVID-19 Pandemics}

Using multiple logistic regression analysis the following variables were found to be significantly associated factors with the psychological impact of the COVID-19 pandemic on students. These were age, gender, residence and students who were involved in the Debre tabor health science college were significantly associated with psychological impact of COVID-19 outbreaks. The odds of being high psychological impact of the COVID-19 among category (18-24years) old participants were 3.6 times more likely than other age's category groups $(\mathrm{AOR}=3.6$; $\mathrm{CI}:(1.140-11.391))$, while age range24-29 years old had 2.70 lower odds of having a high psychological impact of COVID-19 infections (AOR $=0$. $0.37 ; 95 \%$ CI:(0.160-0.852)).

Being male participants had 1.72 times higher odds of having a high psychological impact due to the COVID-19 than female participants (AOR $=1.72 ; 95 \% \mathrm{CI}: 1.037-2.840)$ ) (Table 4).

Table 4. Bivariate and multivariable logistic regression analysis of factors associated with psychological impact of COVID-19 among health science college students, Amhara state of Ethiopia, $2020(N=317)$.

\begin{tabular}{|c|c|c|c|c|}
\hline \multirow{2}{*}{ Variables } & \multicolumn{2}{|c|}{ psychological impacts of COVID-19 } & \multirow{2}{*}{ COR $(95 \% \mathrm{CI})$} & \multirow{2}{*}{ AOR (95\% CI) } \\
\hline & Low & High & & \\
\hline \multicolumn{5}{|l|}{ Ages } \\
\hline $18-24$ & 64 & 70 & $3.68(1.044-11.465)$ & $3.6(1.140-11.391)^{*}$ \\
\hline $25-31$ & 38 & 44 & $0.42(0.159-0.917)$ & $0.37(0.160-1.152)$ \\
\hline $32-38$ & 31 & 33 & $0.66(0.342-2.013)$ & $0.71(0.304-1.639)$ \\
\hline$\geq 39$ & 21 & 16 & 1 & 1 \\
\hline \multicolumn{5}{|l|}{ Sex } \\
\hline Female & 75 & 50 & 1 & 1 \\
\hline Male & 88 & 104 & $1.77(1.138-3.327)$ & $1.72(1.037-2.840)^{*}$ \\
\hline \multicolumn{5}{|l|}{ Religion } \\
\hline Orthodox & 63 & 87 & $0.46(0.225-1372)$ & $0.98(0.393-2.465)$ \\
\hline Muslim & 38 & 41 & $0.59(0.421-3.343$ & $1.0(0.390-1.566$ \\
\hline Protestant & 28 & 19 & $0.94(0.305-1.545)$ & $0.54(0.247-1.199)$ \\
\hline Catholic & 25 & 16 & 1 & 1 \\
\hline \multicolumn{5}{|l|}{ Residence } \\
\hline Rural & 82 & 102 & 1 & 1 \\
\hline Urban & 81 & 52 & $0.52(0.305-0.912)$ & $0.51(0.306-0.838)^{*}$ \\
\hline \multicolumn{5}{|l|}{ Department } \\
\hline Environment & 34 & 27 & 1 & 1 \\
\hline Laboratory & 30 & 37 & $1.55(0.597-2.114)$ & $1.36(0.597-3.089)$ \\
\hline Pharmacist & 22 & 31 & $1.77(0.904-5.245)$ & $2.24(0.939-5.359)$ \\
\hline Nurse & 26 & 18 & $0.87(0.495-3.111)$ & $1.25(0.502-3.113)$ \\
\hline HIT & 19 & 18 & $1.19(0.402-2.742)$ & $1.06(0.394-2.845)$ \\
\hline Health Excitation & 21 & 11 & $0.66(0.335-2.933)$ & $0.93(0.326-2.658)$ \\
\hline Midwife & 11 & 12 & $1.37(0.267-2.674)$ & $0.83(0.265-2.622)$ \\
\hline \multicolumn{5}{|l|}{ Study area } \\
\hline Teda HSC & 59 & 44 & $1.58(0.839-3.172)$ & $1.78(0.973-3.264)$ \\
\hline Debre Tabor HSC & 52 & 53 & $1.16(1.943-7.278)$ & $4.25(2.304-7.842)^{*}$ \\
\hline Bahir Dar HSC & 50 & 59 & 1 & 1 \\
\hline \multicolumn{5}{|l|}{ Family income } \\
\hline$<1500$ & 59 & 51 & $0.74(0.255-1.511)$ & $0.68(0.287-1.626)$ \\
\hline $1500-2500$ & 49 & 49 & $0.64(0.260-1.531)$ & $1.65(0.271-1.551)$ \\
\hline $2501-3500$ & 24 & 49 & $0.31(0.122-0.810)$ & $0.33(0.129-0.826)$ \\
\hline$\geq 3500$ & 22 & 14 & 1 & 1 \\
\hline
\end{tabular}

$\mathrm{HSC}=$ Health Science College, ${ }^{*}$-value $<0.05$

\subsection{Factors Associated with an Early Behavioral Response to COVID-19}

Using multivariable logistic regression analysis, the following variables were found to be significantly associated with an early behavioral response to COVID-19. Gender and itself level of high psychological impact were found to be associated predictors of good early behavioral response to prevent the spread of the COVID-19 infections.
The odds of good early behavioral response to reduceCOVID-19 infection was 3.4 times higher among male students compared with female students (AOR: 3.4; 95\% CI:(1.945-5.137)), and students who had a high psychological impact of COVID-19 were1.9 times more likely to have a good early behavioral response to compact the COVID-19 pandemics (AOR: 1.9; 95\% CI: (1.16-2-96)) (Table 5). 
Table 5. Bivariate and multivariable logistic regression analysis of factors associated with prevents COVID-19 pandemics among health science college students, Amhara regional state, Ethiopia, 2020 (317).

\begin{tabular}{lllll}
\hline \multirow{2}{*}{ Variables } & \multicolumn{2}{l}{ Early behavioral response to COVID-19 } & \multirow{2}{*}{ COR (95\% CI) } & AOR (95\% CI) \\
\cline { 2 - 4 } Age groups & Good & & & \\
$18-24$ & 65 & 69 & $0.89(0.482-3.694)$ & $1.26(0.466-3.424)$ \\
$25-31$ & 40 & 42 & $1.03(571-2.816)$ & $1.23(0.539-2.789)$ \\
$32-38$ & 32 & 32 & $1.00(0.591-3.140)$ & $1.26(0.549-2.899)$ \\
$\geq 39$ & 18 & 19 & & 1 \\
Sex & & & $2.83(2.112-6.077)$ & $3.42(1.945-5.137) *$ \\
Female & 83 & 42 & 1 & 1 \\
Male & 79 & 113 & 1 & 1 \\
Department & & & $1.20(0.623-3.054)$ & $1.31(0.607--2.612)$ \\
Environmental & 31 & 30 & $0.86(0.404-2.107)$ & $0.94(0.433-2.031)$ \\
Laboratory & 31 & 36 & $1.13(0.515-2.975)$ & $1.01(0.482-2.450)$ \\
Pharmacist & 29 & 24 & $1.36(0.688-0.4 .253)$ & $1.48(0.626-3.231)$ \\
Nurse & 21 & 23 & $0.40(0.162-1.233)$ & $0.38(0.140-3.493)$ \\
HIT & 16 & 21 & $1.13(0.311-2.662)$ & $0.98(0.364-2.753)$ \\
Health excitation & 23 & 9 & & \\
Midwife & 11 & 12 & $0.62(0.325-1.167)$ & $0.67(0.355-1.143)$ \\
Study area & & & $0.99(0.529-1.884)$ & $0.95(0.525-1.731)$ \\
TedaHSC & 59 & 44 & 1 & 1 \\
Debre tabor HSC & 53 & 52 & 1 & 1 \\
Bahir Dar HSC & 50 & 59 & $1.52(1.239-3.736)$ & $1.9(1.156-2-964) *$ \\
COVID-19 Psychological impact & & & &
\end{tabular}

\section{Discussion}

The current revealed that theprevalence of psychological impact of COVID-19 and early behavioral responses to COVID-19, and its associated factors among health science college students in Northwest, Amhara regional state since the beginning of the outbreak in Ethiopia in March 13, 2020.

About $51.4 \%$ with $95 \%$ CI $(45 \%, 57 \%)$ of students reported psychological impact of the COVID-19, while $51.6 \%$ with $95 \%$ CI $(46 \%, 57 \%)$ had early behavioral responses to the COVID-19 outbreaks.

The prevalence of the psychological impact of COVID19 on students in this study was higher than in a study conducted in Ethiopia, 44.4\% [32]. This might be due to the currently increase number of COVID-19 cases. For instance, as the Ethiopia ministry of heath reported (August 22/10/2020) pandemicCOVID-19 was a total of 91,693 cases and 1,396 deaths. The other considerable difference might be the use of different tools and method to assess this psychological distress and the different study participants. For instance, a study in that made Google online data collection method was conducted.

The prevalence of early behavioral responses to compact the COVID-19 pandemic in this study was in line with the findings of study conducted in Jimma, Southwest, and Ethiopia [25].

Regarding the demographic characteristics like; 18-24 age, female in gender, urban residence, and students' evolved in study area were significantly associated factors with high psychological impact to the COVID-19 pandemic among students. The odds of psychological impact to the COVID-19 pandemic was almost four times higher among student who found age between 18-24 years old for the high impact of COVID-19pandemic as compared with those student who had a low impact of COVID-19 pandemic. This finding was supported by a study conducted in Australia [41] and in Ethiopia [42] participants who found that young age had a greater psychological impact to COVID-19 pandemic than their counterparts. This result was also similar to a comparison study conducted in Korea, Ethiopia, and Congo showed that poor information about preventive measurements of COVID-19 as compared to counterparts [43]. This might also due to the fact that young and middleaged adults were most at risk and were coping less well with the consequences.

Accordingly, male participants were significantly associated with the high psychological impact of COVID-19 than female participants (AOR $=1.72 ; 95 \%$ CI: (1.0372.840)). This finding is in line with studies conducted in the Netherlands [44] China [27] and Iran [45]. The possible justification might be that majority of males work outside their home by moving from one place to another place. In addition to this, because male students move from place to place more often, they use transportation services, which will be difficult to comply with physical distancing. Therefore, men might be exposed to high stress from COVID-19 outbreaks. On the other hand, in our context, females bear a huge burden of childcare, so they may fear transmitting the disease to their children if they didn't have early behavioral response to compact the COVID-19. Therefore, females might be behavioral response to the national of COVID-19 outbreaks.

This finding also showed that the students who lived in 
urban area were associated with the high psychological impact of COVID-19. Those respondents who live in urban area were 0.51 times less likely to have a high psychological impact on students than those respondents who live in rural areas (AOR $=0.51,95 \%$ CI: $0.306-0.838)$. This study is in line with the study was conducted in Addis Ababa [46]. This might be due to access to information or knowledge differences about the transmission and prevention of COVID-19. Inaddition to this, precautionary measures and psychological readiness for early behavioral response are important to prevent the COVID-19. Hence, when the study participants had high psychological stress from to COVID19 , they were preventive in early response to this outbreak and by adherence by not traveling to crowded places and staying at home.

This study also revealed students who participated in Debre Tabor health science college were 4.25 times more likely to have a high psychological impact of COVID-19 than their counterparts (AOR=4.25, 95\% CI: (2.304-7.842)). The possible explanation for this difference might be related to the pre-information the way how to prevent theCOVID19 pandemics.

Finally, our findings identified that students had high prevalence of psychological impact of COVID-19 was a significant associated factor with early behavioral response to prevent cornea viruses. These findings are in line with the studies conducted in different countries [43, 47, 48]. A possible explanation for this similarity might be the study participants who had a high psychological impact of COVID19 score on the early behavioral response to prevent this viruses were more likely to have an urgent response to prevent COVID-19. The other possible explanation for this anxiety may influence behavioral responses to the belief of being infected.

Limitation

The study acknowledged some important possible limitations that should be considered when interpreting the results. First, the study was cross-sectional, a design that does not permit establishing cause-effect relationships. Second, social desirability and recall bias might be introduced.

\section{Conclusions and Recommendation}

Our findings have indicated that, more than half of the participants were highly psychological impacted by COVID19 and had poor early behavioral response to prevent cornea viruses.

Age, gender, residence, and type of participating study area were significant factors of psychological impact on COVID-19. Gender and participants who had high psychological impact on COVID-19 were also factors in early behavioral response to COVID-19 outbreaks. Therefore, it is crucial to increase mental health awareness, gender-based intervention strategies, and relevant coping mechanisms for students from varied backgrounds that may help the participants cope with the issues related to mental health, during learning. There is a need to develop gender based intervention plan for this psychological problem in the population, mainly targeting those who do not get information from social media and those who did not adequately practice infection prevention and control measures to prevent the COVID-19 pandemic. The government should develop and implement national programs for school based health and safeties, improve psychological well-being, and protect students from physical and mental hazards to take care of the mental health of students during this pandemic.

\section{Declaration}

\section{Ethical Considerations}

Ethical approval was obtained from the Ethical Review Committee of Amhara Public Health Institution (APHI). After an official letter had been submitted to the North Gondar zonal health department's office, permission letters were collected from both the North Gondar zonal health department's office and each health facility office. Informed verbal consent was obtained from the study participants. Names or specific addresses of the study participants were coded and kept anonymous, and confidentiality was assured. Their rights not to participate, not to answer any or all questions, and to withdraw from the interview at any time they want were respected.

\section{Abbreviations}

AOR, Adjusted Odds Ratio; CI, Confidence Interval; COR, Crude Odds Ratio; COVID-19, Corona virus disease 19; PPE, Personal Protective Equipment; SARS, Severe Acute Respiratory Syndrome; SPSS, Statistical Package for Social Sciences; TV, Television; WHO, World Health Organization

\section{Consent for Publication}

Not applicable.

\section{Availability of Data and Materials}

The dataset analyzed during the current study is available from the corresponding author on reasonable request.

\section{Conflict of Interest Statement}

The authors declare that they have no competing interests

\section{Authors' Contributions}

AM- was involved in the conception and design of the research project proposal, collect, analyzes, interpret the data and results and preparation and critical review of the manuscript. Other author (GM) was involved in reviewing the research project proposal and interpretation of results as well as preparation and critical review of the manuscript. 


\section{Acknowledgements}

Our sincere thanks also go to Amhara public health institute for giving us ethical clearance and approval. We are very indebted to the college deans, catchment study area for permitting to conduct the study and for providing the necessary preliminary information while conducting this study. We do wish to extend our gratitude to the study participants, supervisors and data collectors. Finally, we sincerely appreciate our friends for supporting us in writing and reviewing the manuscript.

\section{References}

[1] WHO: Critical preparedness, readiness and response actions for COVID-19: interim guidance World Health Organization 2020.

[2] Wang C HP, Hayden FG, Gao GF: A novel coronavirus outbreak of global health concern Lancet https://doiorg/10 1016/S0140-6736(20)30185-9 2020.

[3] CN EaM: Sub-Saharan Africa prepared for COVID-19? Tropical Medicine and Health 2020, 48 (1): 1-3.

[4] Morens DM FA: Emerging infectious diseases: threats to human health and global stability. PLoS Pathog https://doiorg/101371/journalppat1003467 PMID: 23853589 2013, 9 (7) (e1003467).

[5] Solomou I CF: Prevalence and predictors of anxiety and depression symptoms during the COVID-19 pandemic and compliance with precautionary measures: age and sex matter. International journal of environmental research and public health https://doiorg/103390 2020, 17 (14): 4924.

[6] Kshipra Moghe DK, Manjusha Patil: COVID-19 and Mental Health Impact on https://doiorg/101101/2020080520160499 2020.

[7] WHO: WHO Coronavirus Disease (COVID-19) Available from: https://COVID19whoint/?gclid=Cj0KCQiAhs79BRD0ARIsA C6XpaVKVf1AmzsG1TR-

1m6f5PGCB1KztaxrroLTtyE3Zr9KJ-

uIe8iOkwaAhRTEALw_wcB 2020.

[8] Sisay Teklu MS, Aklilu Azazh, etail: Clinical and Sociodemographic Profile of the First 33 COVID-19 Cases Treated at Dedicated Treatment Center in Ethiopia. Ethiop J Health Sci 2020; 30 (5): 645 doi: http://dxdoiorg/104314/ejhsv30i52 2020.

[9] Olapegba PO AO, Kolawole SO, etail: Preliminary Assessment of Novel Coronavirus (COVID-19) Knowledge and Perceptions in Nigeria. medRxiv. medRxiv2020.

[10] CPERE N: The epidemiological characteristics of an outbreak of 2019 novel coronavirus diseases (COVID-19) in China. Zhonghua liu xing bing xue za zhi=Zhonghua liuxingbingxue zazhi. https://doiorg/103760/cmajissn0254-6450202002003 PMID 2020, 41 (2): 145 (32064853).

[11] COVID-19 CX: immunopathology and its implications for therapy. Nature reviews immunology https://doiorg/101038/s41577-020-0308-3 PMID: 32273594 2020, 20 (5): 269-270.
[12] KH: Economic impacts of COVID-19 pandemic in Ethiopia: A review of phone survey evidence 2020.

[13] Moukaddam N SAPB: The impact of COVID-19 and pandemics on mental health. Psychiatric times. https://wwwpsych iatric crime scom/psych iatri sts-bewar eimpac t-coron aviru s-pande micsmental-health 2020.

[14] WHO: Coronavirus Disease 2019 (COVID-19) Situation Report 2019, 60.

[15] Verso MAC: Climate, Chronic Emergency. War Communism in the Twenty-First Century 2020.

[16] Zelalem Nigussie Azene MWM, Atalay Goshu Muluneh, etail: Adherence towards COVID-19 mitigation measures and its associated factors among Gondar City residents: A community-based cross-sectional study in Northwest Ethiopia. PLOS ONE | https://doiorg/101371/journalpone0244265 December 30, 2020.

[17] Cascella M RM, Cuomo A, Dulebohn SC, Di Napoli R: Features, evaluation and treatment coronavirus (COVID-19). Stat Pearls [Internet] 2020.

[18] Brooks SK WR, Smith LE, et al: The psychological impact of quarantine and how to reduce it: rapid review of the evidence Lancet 2020; 395 (10227): 912-920 doi: 101016/S01406736(20)30460-8 2020.

[19] Shigemura J UR, Morganstein JC, Kurosawa M, Benedek DM: Public responses to the novel 2019 coronavirus (2019$\mathrm{nCoV}$ ) in Japan: mental health consequences and target populations. Psychiatry Clin Neurosci 2020; 74 (4): 281 doi: 101111/pcn12988 2020.

[20] EP: COVID-19 threatens health systems in sub-Saharan Africa: the eye of the crocodile". The clinical investigation 2020.

[21] Que J LSJ, Liu J, et al: Psychological impact of the COVID19 pandemic on healthcare workers: a cross-sectional study in China Gen Psychiatry doi: 101136/gpsych-2020-100259 2020, 33: 3 .

[22] Tesfaye Kebede MH: Impact of COVID-19 pandemic on radiology residency training in ETHIOPIA. Ethiop Med J 2020, 58, No. 4.

[23] Francesco Di Gennaro DP, Claudia Marotta, etail: Coronavirus Diseases (COVID-19) Current Status and Future Perspectives. Int J Environ Res Public Health 2020.

[24] Niu Y XF: Deciphering the power of isolation in controlling COVID-19 outbreaks. The Lancet Global Health 2020, 8 (e452-3).

[25] Yohannes Kebede YY, Zewdie Birhanu, Seblework Mekonen, Argaw Ambelu: Knowledge, perceptions and preventive practices towards COVID-19 early in the outbreak among Jimma university medical center visitors, Southwest Ethiopia. plos one 2020 .

[26] Lake J TM: Urgent need for improved mental health care and a more collaborative model of care Perm J 2017, 21: 17-024.

[27] Erfani A SR, Ranjbar K, Mirahmadizadeh A, Moghadami M: Knowledge, Attitude and Practice toward the Novel Coronavirus (COVID-19) Outbreak A Population-Based Survey in Iran. https://wwwwhoint/bulletin/online_first/20256651Bull World Heal Organ 2020. 
[28] DFT L: Understanding and Addressing Sources of Anxiety Among Health Care Professionals During the COVID-19 Pandemic.

[29] JG: The COVID-19 crisis too few are talking about: health care workers' mental health Stat Reports https://wwwstatn ewscom/2020/04/03/the-COVID-19-crisi s-too-few-are-talki ng-about -healt h-care-worke rs-mental-healthAccessed 10 Apr 2020.

[30] Matryna plomecka pr: Mental health impact of COVID-19. Researchgate 2020.

[31] Akaninyene Otu CHC, and Sanni Yaya: Mental health and psychosocial well-being during the COVID-19 pandemic: The invisible elephant in the room. BMC Mental Health Systems 2020.

[32] Argaw Ambelu ZB, Yimenu Yitayih, etail: Psychological Distress During the COVID-19 Pandemic in Ethiopia. Research square 2020.

[33] Barry Bogin CV, School of Sport, Exercise and Health Sciences, Loughborough: COVID-19, crisis, and emotional stress: A biocultural perspective of their impact on growth and development for the next generation. Am J Hum Biol 2020.

[34] Wudneh Simegn BD, Yigizie Yeshaw, Sewbesew Yitayih: Depression, anxiety, stress and their associated factors among Ethiopian University students during an early stage of COVID-19 pandemic: An online-based cross-sectional survey. ploS ONE 2021.

[35] Odriozola-Gonza' lez P P-Gm, Irurtia MJ, de Luis-Garc1'a R: Psychological effects of the COVID-19 outbreak and lockdown among students and workers of a Spanish university. Psychiatry Research https://doiorg/101016/jpsychres2020113108 PMID: 32450409 2020 (113108).

[36] Cao W FZ, Hou G, Han M, Xu X, Dong J, et al: The psychological impact of the COVID-19 epidemic on college students in China. Psychiatry research https://doiorg/101016/jpsychres112934 PMID: 32229390 2020 (112934).

[37] Dagnew B DH, Andualem Z: Depression and Its Determinant Factors Among University of Gondar Medical and Health Science Students, Northwest Ethiopia: Institution-Based Cross-SectionalStudy. Neuropsychiatric Disease and Treatmenthttps://doiorg/102147/NDTS248409 PMID: 32280225 2020, 16: 839 .

[38] Chang J YY, Wang D: Mental health status and its influencing factors among college students during the epidemic of COVID-19. Nan fang yikedaxuexuebao=Journal of Southern
Medical University. https://doiorg/1012122/jissn1673425420200206 PMID: 32376528 2020, 40 (2): 171.

[39] Semrau M AA, Ayuso-Mateos JL, Chisholm D, Gureje O, Hanlon C, et al: Strengthening mental health systems in lowand middle-income countries: recommendations from the Emerald programme. BJPsych Open: e73 https://doiorg/101192/bjo2018 2019, 5 (5): 90.

[40] WHOb: Survey Tool and Guidance: Rapid, Simple, Flexible Behavioural Insights on COVID-19. DK-2100 Copenhagen, Denmark 2020.

[41] Taylor MR AK, Stevens GJ, Raphael B: Factors infuencing psychological distress during a disease epidemic: Data from Australia's first outbreak of equine influenza. BMC Public Health 2008, 8: 347.

[42] Atkure Defar, Gebeyaw Molla, Saro Abdella: Knowledge, Practice and associated factors towards the Prevention of COVID-19 among high-risk groups: A cross-sectional study in Addis Ababa, Ethiopia. https://doiorg/101101/2020081420172429 2020.

[43] Hocheol Lee SJM, Grace Ossak Ndombi, etail: COVID-19 Perception, Knowledge, and Preventive Practice: Comparison between South Korea, Ethiopia, and Democratic Republic of Congo. DOI: 1029063/ajrh2020/v24i2s11 2020.

[44] Nivette A RD, Murray AL, Steinhoff A, Bechtiger L, Hepp U, et al: Non-compliance with COVID-19-related public health measures among young adults: Insights from a longitudinal cohort study 2020.

[45] Zhong BL, LiH, Zhang Q, LiuX, LiW, etal: Knowledge, attitudes, and practices towards COVID-19 among Chinese residents during the rapid rise period of the COVID-19 outbreak. Int J Biol Sci 2020, 16 (10): 1745-1752.

[46] Abebe Habtamu Tamire TKL: knowledge, attitudes, and practices towards coronavirus disease-19 among health professionals in addis ababa. Ethiop Med J 2020, 58 (4).

[47] Iao H TN, Han N, Khanh T Van, Ngan VK, Tam V Van, et al: Knowledge and attitude toward COVID-19 among healthcare workers at Knowledge and attitude toward COVID-19 among healthcare workers at District 2 Hospital, Ho Chi Minh City. Asian pac J Trop Med 2020, 13 (April): 1-7.

[48] Konstantinos Kontoangelos, Marina Econo, Charalambos Papageorgiou: Mental Health Effects of COVID-19 Pandemia: A Review of Clinical and Psychological Traits. Neuropsychiatric Association 2020. 